\title{
Inhibitory control and the adolescent brain: a review of fMRI research
}

\author{
Antonio Jaeger \\ Universidade de São Paulo, São Paulo, SP, Brazil
}

\begin{abstract}
Adolescence is a developmental period frequently characterized by impulsive behavior and suboptimal decision making, aspects that often result in increased rates of substance abuse, unprotected sex, and several other harmful behaviors. Functional magnetic resonance imaging (fMRI) studies have attempted to reveal the brain mechanisms that underlie the typical inhibitory control limitations associated with this developmental period. In the present review, all available studies in the PsycINFO, PubMed, and Web of Science databases that investigated this issue utilizing fMRI were analyzed. In contrast to adults, adolescents exhibited decreased activity in several brain regions associated with inhibitory control such as the dorsolateral prefrontal cortex, anterior cingulate cortex, and fronto-striatal regions. The decreased activity found in these regions may underlie the diminished inhibitory control abilities associated with this development period.
\end{abstract}

Keywords: inhibitory control, development, adolescence, fMRI, anti-saccade, Go/No-go.

Received 8 January 2013; received in revised form 4 March 2013; accepted 5 March 2013. Available online 27 June 2013.

\section{Introduction}

During adolescence, important neurodevelopmental processes such as myelination and gray matter pruning still take place in regions typically involved in cognitive control (Gogtay et al., 2004). The immaturity of these regions is thought to underlie the suboptimal decision making and actions that are typically encountered in this population (Casey \& Jones, 2010), which can ultimately result in increased risky and harmful behaviors such as experimentation with drugs and criminal activity (Eaton et al., 2006). Adolescence is also a period during which the symptoms of major psychiatric disorders such as schizophrenia and attention-deficit/ hyperactivity disorder begin to manifest (Insel, 2010). Thus, understanding the neurobiological aspects of the adolescent brain may provide a better understanding of healthy adolescents, and help to provide potential treatments for neural and psychological disorders typically associated with this developmental period (Casey \& Jones, 2010).

One aspect often implicated in the onset and maintenance of certain mental disorders during adolescence is the ability to suppress the cognitive processing of undesired information (Luna, Padmanabhan, \& O'Hearn,

Antonio Jaeger, Universidade de São Paulo. Correspondence regarding this article should be directed to: Antonio Jaeger, Universidade de São Paulo/USP, Instituto de Biociências, Departamento de Fisiologia Geral, Rua do Matão, Travessa 14, 101, Cidade Universitária, São Paulo, SP, CEP 05508900, Brasil. Phone: (11) 3091-7504. E-mail: antonio.jaeger@ gmail.com
2010), a cognitive function often referred to as inhibitory control (Miller, 2000). Prior behavioral studies showed that adolescents differ from adults in their inhibitory control abilities (e.g., Luna, Garver, Urban, Lazar, \& Sweeney, 2004). These differences are usually attributed to the protracted development of brain regions that may be necessary for the full operation of this function. As shown by prior research (Gogtay et al., 2004), myelination and gray matter pruning processes are still ongoing during adolescence and early adulthood in prefrontal regions that are often engaged in tasks that require a certain level of inhibitory control (Ridderinkhof, Ullsperger, Crone, \& Nieuwenhuis, 2004; Badre \& Wagner, 2004).

Specifically in adults, functional magnetic resonance imaging (fMRI) studies that have investigated inhibitory control suggest critical roles for the dorsolateral prefrontal cortex and anterior cingulate cortex. The dorsolateral prefrontal cortex is thought to be involved in the implementation of control (MacDonald, Cohen, Stenger, \& Carter, 2000), whereas the anterior cingulate cortex is often engaged during conflict resolution and error monitoring (Botvinick, Braver, Barch, Carter, \& Cohen, 2001). Parietal areas also appear to be relevant for inhibitory control in adults (Garavan, Ross, \& Stein, 1999), apparently by supporting attentional processes that enable the implementation of inhibitory control (Corbetta \& Shulman, 2002). Motivated perhaps by the implications of the development of inhibitory control for overall mental health, accumulating neuroimaging research has investigated the brain regions that are differentially activated in adolescents compared with adults when inhibitory control is exerted (e.g., Velanova, Wheeler, 
\& Luna, 2009). Research on such a topic can potentially clarify, for example, whether the suboptimal behavior often presented by adolescents is caused by impaired error monitoring and diminished engagement of the anterior cingulate cortex or whether potential functional alterations of the dorsolateral cortex are associated with difficulties preparing for and implementing inhibitory control during this developmental period.

Thus, the goal of the present article is to expand the understanding of the functional aspects of the healthy adolescent brain that underlie the diminished inhibitory control capacities typically found in this population. To achieve this, a review was conducted of fMRI studies that investigated inhibitory control in healthy adolescents. Reports of fMRI studies that investigated inhibitory control in healthy adolescents that are indexed in the PubMed, PsycINFO, and Web of Sciences databases are covered in the present review. To facilitate the comprehension of the procedures adopted by the studies covered herein, the Results section of the present article is divided into subsections according to the behavioral task utilized to engage inhibitory control. Studies that employed anti-saccade tasks are discussed first followed by studies that employed Go/No-Go tasks and other experimental manipulations.

\section{Methods}

A literature search was conducted by selecting articles from the Web of Science, PubMed, and PsycINFO databases that reported fMRI experiments that manipulated inhibitory control in adolescents. The search was performed in July 2012 and updated in December 2012 using the keywords "inhibitorycontrol" or "response-inhibition" in combination with the keywords "fMRI" and "adolescents." To find potentially relevant studies that were not indexed in these databases, searches of the reference lists of the selected articles were performed after selecting articles from the databases.

The inclusion criteria were the following: (1) the article must be published in a peer-reviewed journal, (2) the article must report experiments in which inhibitory control was investigated in adolescents using behavioral tasks and brain activity monitoring by fMRI, and (3) only healthy participants were studied (i.e., non-clinical sample). Articles that reported pharmacological studies and manuscripts that did not meet the aforementioned inclusion criteria were excluded.

\section{Results}

From the initial database search, 361 articles were found using the aforementioned keyword combinations. Of these, 159 were found in PubMed, 163 were found in Web of Science and 39 were found in PsycINFO. After eliminating duplicate manuscripts, the articles were selected based on the inclusion and exclusion criteria mentioned above, yielding 11 papers for the final analysis. The searches of the reference lists did not yield articles that were not already included from the database search.

\section{Anti-saccade task}

In the anti-saccade task, participants initiate each trial by looking at a fixation point in the center of a computer screen. They are subsequently required to look at the opposite side (i.e., mirror position) of a target that can appear on either the left or right side of the fixation point. To perform this task, the participants must first inhibit the automatic response of looking in the target direction (i.e., pro-saccade) and then convert the input of the target's location using a motor command to look in the opposite direction from the target (i.e., antisaccade; Munoz \& Everling, 2004). To examine antisaccade inhibitory responses, anti-saccade performance is often contrasted with performance on a pro-saccade task (i.e., the participants are instructed to look at the target stimulus). Eye tracking equipment is utilized to monitor eye movements, enabling the recording of response accuracy and response time, which are usually the dependent variables in studies that utilize this paradigm.

Neuroimaging and neurophysiological findings from human and primate studies demonstrated that certain brain regions such as lateral parietal areas, superior colliculus, frontal eye fields, supplementary eye fields, and the dorsolateral prefrontal cortex are critical for prosaccade and anti-saccade processing and performance in adults. More specifically, lateral parietal regions appear to represent an interface between sensory and motor processing (Colby \& Goldberg, 1999). The superior colliculus plays a role in the generation of saccadic activity by integrating exogenous and endogenous inputs (Trappenberg, Dorris, Munoz, \& Klein, 2001). The frontal eye field is critical for the motor execution of voluntary saccades (Pierrot-Deseilligny, Rivaud, Gaymard, Muri, \& Vermersch, 1995). The supplementary eye field plays a role in monitoring the context and consequence of oculomotor movements (Stuphorn, Taylor, \& Schall, 2000). The dorsolateral prefrontal cortex plays an important role in the preparation of anti-saccade movements, inhibition of automatic prosaccade responses, and decision processes that support oculomotor movements (Pierrot-Deseilligny, Muri, Nyffeler, \& Milea, 2006; Munoz \& Everling, 2004).

Prior behavioral studies demonstrated that behavioral performance on this task differs according to age (Fischer, Biscaldi, \& Gezeck, 1997). Children exhibit slower reaction times and make more errors in the anti-saccade task than adolescents and adults. Adult-like performance on these measures appears to be reached during midadolescence (Munoz, Broughton, Goldring, \& Armstrong, 1998), although some improvements are still found until 25 years of age (Fischer et al., 1997), presumably reflecting the protracted development of regions that are intrinsically related to inhibitory control (Gogtay et al., 2004). These findings are consistent with the characterization of maturity as not simply having the ability to perform a task but also performing a task at adult-like levels. Children are already capable of performing response inhibition tasks, 
but these tasks are not mastered until adolescence (Luna \& Sweeney, 2004).

In an early attempt to investigate the brain correlates of differences in inhibitory control between age groups, Luna et al. (2001) utilized fMRI to examine the brain regions that were differentially activated during the performance of an anti-saccade task in three age groups: 8-13 years old $(11$ children; mean age $=10.9$ years; standard deviation $[\mathrm{SD}]=1.5$ years; eight females), $14-17$ years old ( 15 adolescents; mean age $=15.7$ years; $\mathrm{SD}=1.2$ years; six females), and 18-30 years old (10 young adults; mean age $=24.2$ years; $\mathrm{SD}=2.9$ years; six females). These authors found that adults showed more activated voxels and a higher percent signal change during the correct performance of anti-saccade trials compared with the other age groups in the superior frontal eye field, lateral cerebellum, and superior colliculus. Adolescents showed more activated voxels and a higher percent signal change than the other age groups in the inferior frontal eye field, pre-supplementary motor area, and right dorsolateral prefrontal cortex (Table 1).

Notably, these authors found differences in hemodynamic activation as a function of age in brain regions that are typically associated with oculomotor control such as the frontal eye field and supplementary motor area (Pierrot et al., 2006; O'Driscoll, Alpert, Matthysse, Levy, Rauch, \& Holzman, 1995). The experimental design used by these authors, however, hindered any strong interpretations of these findings. They used a blocked design (i.e., experimental blocks with only one experimental condition each) instead of event-related designs (i.e., experimental blocks with all conditions intermixed). The main problem with results from such a design is perhaps the impossibility of separating the brain activation elicited by correct antisaccade responses from the brain activity elicited by incorrect anti-saccade responses, precluding the analysis of "pure" anti-saccade responses (Henson, 2006).

In a more recent study, Velanova, Wheeler, \& Luna (2008) used an anti-saccade task in participants with similar ages as the participants included in the study reported by Luna et al. (2001): 18-27 years old (28 adults; mean age $=20.8$ years; $\mathrm{SD}=2.79$ years), $13-17$ years old ( 35 adolescents; mean age $=15.32$ years; $\mathrm{SD}=1.63$ years), and $8-12$ years old ( 35 children; mean age $=10.50$ years; $\mathrm{SD}=1.39$ years). No significant differences in IQ were found across groups as measured by the Wechsler Abbreviated Scale of Intelligence (WASI; Wechsler, 1999). The data analysis, however, utilized an eventrelated design, allowing the opportunity to separately analyze brain activity elicited by correct and incorrect anti-saccade responses (Henson, 2006). Although this study showed that regions typically associated with oculomotor control had greater activation when participants performed correct anti-saccade responses, no differences as a function of age were found. The engagement of oculomotor regions such as the frontal eye field and parietal regions increased similarly in all age groups when their responses were correct.
Interestingly, correct anti-saccade trials elicited greater engagement of the dorsal anterior cingulate cortex in adults than in adolescents and children. Prior research suggested that this region is important for error processing (Polli, Barton, Cain, Thakkar, Rauch, \& Manoach, 2005), and increased activation of this region as a function of age was interpreted as an improvement in error control function (i.e., error-regulatory function). Furthermore, this study showed that a shift occurs during development from predominantly frontal activity to predominantly posterior activity during anti-saccade responses. These results suggest that the improvement in inhibitory control during adolescence results from the augmented engagement of the anterior cingulate cortex for error control and involvement of posterior regions to support attentional and sensory processing (Table 1).

Similar to inhibitory control, reward processing is an important function that can underlie impulsive behavior in adolescents. Prior research showed that regions that support cognitive control and higher order processing remain immature during adolescence, but reward-related regions appear to be well-developed during this period (Casey, Getz, \& Galvan, 2008). An important question is how regions that support these processes interact in a task that engages inhibitory control and reward. Another question is how reward can influence inhibitory control. To examine these issues, Geier, Terwilliger, Teslovich, Velanova, \& Luna (2010) conducted an experiment in which 18 adolescents (13-17 years old; mean age $=15.3$ years; eight females) and 16 young adults (18-30 years old; mean age $=21.7$ years; 10 females $)$ performed an anti-saccade task in which reward was manipulated probabilistically. More specifically, before each anti-saccade trial, a cue indicated whether a monetary reward would be given in the case of a correct response. This manipulation allowed the researchers to examine whether developmental differences existed in the anti-saccade trials when the reward was given and investigate brain activity during different stages of reward processing such as the processing of incentive cues and response preparation.

Behaviorally, both groups were faster and made more correct anti-saccade responses in rewarded trials compared with neutral trials, although this difference reached significance in the adolescent group only. During the processing of incentive cues presented at the onset of each trial indicating whether the trial was rewarded, adults showed more positive activity in the ventral striatum than adolescents when the trials were rewarded. When the preparation of responses was analyzed (i.e., a blank screen that preceded the anti-saccade task by $1500 \mathrm{~ms}$ ), adolescents exhibited heightened activation in ventral striatum during the rewarded trials than during the neutral trials. Adults exhibited reduced activity during reward trials in this same region (Table 1). Overall, Geier et al. (2010) demonstrated that when reward is provided during the performance of an inhibitory control task, adolescents exhibit reduced activity compared with adults in the 
Table 1. Brief description of the experiments reviewed in the present study

\begin{tabular}{|c|c|c|c|}
\hline Study & Task & Group/Age & Key Findings \\
\hline Luna et al., 2001 & Anti-saccade & $\begin{array}{l}11 \text { children, } 15 \\
\text { adolescents, } 10 \text { adults }\end{array}$ & $\begin{array}{l}\text { Adults }>\text { Adolescents: superior frontal eye field, lateral cerebellum, and } \\
\text { superior colliculus } \\
\text { Adolescents }>\text { Adults: inferior frontal eye field, pre-supplementary motor } \\
\text { area, and right dorsolateral prefrontal cortex }\end{array}$ \\
\hline $\begin{array}{l}\text { Velanova et al., } \\
2008\end{array}$ & Anti-saccade & $\begin{array}{l}35 \text { children, } 35 \\
\text { adolescents, } 28 \text { adults }\end{array}$ & Adults $>$ Adolescents: dorsal anterior cingulate cortex \\
\hline Geier et al., 2010 & $\begin{array}{l}\text { Anti-saccade } \\
\text { rewarded }\end{array}$ & 18 adolescents, 16 adults & $\begin{array}{l}\text { Adults }>\text { Adolescents: ventral striatum during processing of incentive cues } \\
\text { Adolescents }>\text { Adults: ventral striatum during response preparation }\end{array}$ \\
\hline $\begin{array}{l}\text { Padmanabhan } \\
\text { et al., } 2011\end{array}$ & $\begin{array}{l}\text { Anti-saccade } \\
\text { rewarded }\end{array}$ & $\begin{array}{l}10 \text { children, } 10 \\
\text { adolescents, } 10 \text { adults }\end{array}$ & $\begin{array}{l}\text { Adolescents }>\text { Adults: right inferior parietal sulcus, bilateral putamen, and } \\
\text { bilateral ventral striatum during rewarded trials compared with neutral } \\
\text { trials }\end{array}$ \\
\hline $\begin{array}{l}\text { Velanova et al., } \\
2009\end{array}$ & Anti-saccade & $\begin{array}{l}26 \text { children, } 25 \\
\text { adolescents, } 27 \text { adults }\end{array}$ & $\begin{array}{l}\text { Adults }>\text { Adolescents: right dorsolateral prefrontal cortex, left anterior } \\
\text { prefrontal cortex, right superior temporal/parietal cortex, and bilateral } \\
\text { occipital regions }\end{array}$ \\
\hline Hwang et al., 2010 & Anti-saccade & $\begin{array}{l}26 \text { children, } 25 \\
\text { adolescents, } 27 \text { adults }\end{array}$ & $\begin{array}{l}\text { Adults }>\text { Adolescents: strength and number of top-down connections from } \\
\text { frontal regions to cortical and subcortical regions }\end{array}$ \\
\hline Tamm et al., 2002 & Go/No-go & $\begin{array}{l}19 \text { participants }(8-20 \\
\text { years old })\end{array}$ & $\begin{array}{l}\text { Age-related increase in activation in the left inferior frontal gyrus/insula } \\
\text { area and orbitofrontal gyrus }\end{array}$ \\
\hline Stevens et al., 2007 & Go/No-go & 25 adolescents, 25 adults & $\begin{array}{l}\text { Adults>Adolescents: integration between regions of the fronto-striatal- } \\
\text { thalamic network }\end{array}$ \\
\hline Rubia et al., 2006 & Go/No-go & 23 adolescents, 25 adults & $\begin{array}{l}\text { Adults }>\text { Adolescents: fronto-striatal regions, including anterior cingulate } \\
\text { gyrus and caudate }\end{array}$ \\
\hline $\begin{array}{l}\text { Adleman et al., } \\
2002\end{array}$ & Stroop & 11 adolescents, 11 adults & Adults $>$ Adolescents: left middle frontal gyrus \\
\hline Rubia et al., 2007 & Stop-signal & 26 adolescents, 21 adults & $\begin{array}{l}\text { Adults }>\text { Adolescents: right inferior prefrontal cortex during successful } \\
\text { inhibition and rostral anterior cingulate gyrus during inhibition failure } \\
\text { Adolescents }>\text { Adults: bilateral insula, left thalamus, putamen, and } \\
\text { posterior cingulate gyrus }\end{array}$ \\
\hline
\end{tabular}

Adults $>$ Adolescents indicates regions that are more active in adults than adolescents. Adolescents $>$ Adults indicates regions that are more active in adolescents than adults.

ventral striatum when initially processing the incentive cues. During response preparation, however, this pattern was reversed (i.e., adolescents exhibited enhanced activity in the ventral striatum compared with adults). Given that the ventral striatum is heavily involved in reward processing, this finding can be interpreted as reflecting weaknesses in the initial assessment of reward and increased reactivity to the anticipation of reward in adolescents compared with adults.

Although Geier et al. (2010) demonstrated that regions that support reward in an inhibitory control task remain immature during adolescence, they did not determine whether these immaturities found in adolescents are similarly exhibited by children. To examine this possibility, Padmanabhan, Geier, Ordaz, Teslovich, \& Luna (2011) administered the same task developed by Geier et al. (2010) in 10 adults (18-25 years old; mean age $=20.6$ years; $\mathrm{SD}=2.2$ years; six females), 10 adolescents $(14-17$ years old; mean age $=$ 15.8 years; $\mathrm{SD}=1.2$ years; six females), and 10 children $(8-13$ years old; mean age $=11.1$ years; $\mathrm{SD}=1.5$ years; six females). No significant differences in IQ were found across groups measured by the WASI (Wechsler, 1999). Behaviorally, these authors showed that although both children and adolescents exhibited inferior performance compared with adults when the trials were not rewarded (i.e., neutral trials), they reached adult-like performance when reward was added. In contrast to Geier et al. (2010), no separate neuroimaging analyses of preparation, incentive, and anti-saccade responses were performed. These authors simply contrasted blood oxygen leveldependent (BOLD) responses for all rewarded and nonrewarded anti-saccade trials. All age groups engaged oculomotor control regions such as the frontal eye field, supplementary eye field, inferior parietal sulcus, parietal regions, and dorsal anterior cingulate cortex. Similarly, regions involved in reward were also activated across ages such as the ventral striatum, orbitofrontal cortex, and anterior cingulate cortex (Table 1), suggesting that the fundamental circuitry that sustains reward and inhibitory control is already developed during childhood and adolescence.

In contrast to adults and children, adolescents exhibited enhanced BOLD responses during rewarded trials compared with neutral trials across the right inferior parietal sulcus, bilateral putamen, and bilateral ventral striatum. As suggested by Padmanabhan et al. (2011), the enhanced activity in the inferior parietal sulcus and putamen during rewarded trials indicates that these regions actually support the improvement in 
performance in these trials and have been previously associated with oculomotor control, response planning (Everling \& Munoz, 2000), and reward processing (Delgado, Locke, Stenger, \& Fiez, 2003). The authors also suggested that ventral striatal activity may underlie the tendency of adolescents to favor immediate over delayed rewards because this region is heavily associated with various aspects of reward processing (e.g., Bjork, Knutson, Fong, Caggiano, Bennett, \& Hommer, 2004).

The studies discussed above found differences between age groups in regions involved in the performance of anti-saccade tasks, but they did not investigate whether these differences reflect transient trial-by-trial activations or activations that persist during the entire task. Velanova et al. (2009) investigated this issue by studying three age groups: $8-12$ years old (26 children; mean age $=10.5$ years; $\mathrm{SD}=1.4$ years), $13-17$ years old (25 adolescents; mean age $=15.3$ years; $\mathrm{SD}=$ 1.6 years), and $18-27$ years old ( 27 adults; mean age $=$ 20.7 years; $\mathrm{SD}=2.7$ years). No significant differences in IQ were found between adolescents and adults as measured by the WASI (Wechsler, 1999). These authors found that some of the regions that exhibited sustained effects also exhibited increased activation during development. These regions consisted of the right dorsolateral prefrontal cortex, left anterior prefrontal cortex, right superior temporal/parietal cortex, and bilateral occipital regions. The authors suggested that the protracted development of these regions results in suboptimal sustained inhibitory control during adolescence.

To investigate the effective connectivity (i.e., direct influences between neural populations) that support inhibitory control across development, Hwang, Velanova, \& Luna (2010) analyzed the data previously reported by Velanova et al. (2009) using Granger causality analysis (Roebroeck, Formisano, \& Goebel, 2005). These authors found an increase in the strength and number of top-down connections from frontal regions to other cortical and subcortical regions from adolescence to adulthood. As suggested by the authors, these increases in frontal top-down effective connectivity may support the improvement in inhibitory control across development (Hwang et al., 2010).

Overall, studies that utilized anti-saccade tasks to investigate inhibitory control in adolescents found that adolescents exhibited decreased activation in the dorsal anterior cingulate (Velanova et al., 2008) and dorsolateral prefrontal cortices (Velanova et al., 2009; Luna et al., 2001) compared with adults, a finding that presumably reflects their diminished capacity of error monitoring and task implementation, respectively. When reward was provided during the performance of the anti-saccade task (Geier et al., 2010; Padmanabhan et al., 2011), adolescents exhibited reduced ventral striatum activity compared with adults during the initial processing of incentive cues but exhibited enhanced activity in this region compared with adults during the latter preparation of responses. These findings can be interpreted as neural evidence of the adolescents' limitations in the assessment of reward and enhanced reactivity to the anticipation of reward (Geier et al., 2010). Although differences across ages in regions involved in the motor execution of saccadic movement were not evident, with the exception of the study that adopted a blocked design (Luna et al., 2001), Granger causality analysis demonstrated an increase in effective connections from frontal regions to other cortical and subcortical regions during development (Hwang et al., 2010). This aspect, in conjunction with the heightened functionality of the dorsolateral and dorsal anterior cingulate cortices, may underlie the inhibitory control advantages that are typically found in adults compared with adolescents.

\section{Go/No-go task}

The Go/No-go task consists of the presentation of a series of stimuli. For a given stimulus type, the participants are required to make a motor response (Go). For another stimulus type, the participants are required to withhold a motor response (No-go; Watanabe et al., 2002). Trials that require a Go response are typically more frequent than trials that require a No-go response. Accuracy and reaction time are recorded for each response type. Brain responses to this task have been widely investigated using both event-related potentials (ERPs) and fMRI (Falkenstein, Hoorman, \& Hohnsbein, 1999; Simmonds, Pekar, \& Mostofsky, 2008), suggesting that the No-go trials in this task elicit brain activity that supports inhibitory control (c.f., Nieuwenhuis, Yeung, van den Wildenberg, \& Ridderinkhof, 2003). Specifically, ERP studies have typically shown that this task (No-go trials) elicits early negative effects ( $200 \mathrm{~ms}$ poststimulus onset) distributed over frontally located electrodes, an ERP component named N200 (Luck, 2005). Recent ERP findings suggested that this negative effect is generated by neural activity in the left anterior region of the mid-cingulate cortex (Huster, Westerhausen, Pantev, \& Konrad, 2010). FMRI research, however, indicates that several other regions also appear to be activated during No-go trials such as ventrolateral and dorsolateral prefrontal regions (Liddle, Kiehl, \& Smith, 2001) and posterior intraparietal and occipitotemporal areas (Watanabe et al., 2002).

In an early attempt to determine whether the involvement of regions that are engaged during the $\mathrm{Go} /$ No-go task is modified during development, Tamm, Menon, \& Reiss (2002) studied 19 participants (8-20 years old; mean age $=14.4$ years; $\mathrm{SD}=3.1$ years; 11 females) who performed a Go/No-go task in the MRI scanner. Cognitive function was assessed using the Wechsler Intelligence Scale for Children (WISC-III) and Wechsler Adult Intelligence Scale (WAIS-III). Between-group IQ differences were not reported in the manuscript. Specifically in their Go/No-go task, a series of letters were presented $(2 \mathrm{~s}$ each). The participants pressed a key in response to every letter except the letter "X." In the Go block, the participants were presented 
a series of letters except the letter "X." In the No-go block (i.e., experimental block), the participants were presented the letter " $X$ " during half of the trials, thus requiring the emission of responses during half of the trials and suppression of responses in the other half (i.e., when an " $X$ " was shown). Behaviorally, no accuracy differences were found across development, although response times decreased with age. When the Go/No-Go blocks were contrasted with the Go blocks, age-related increases in activation were found in the left inferior frontal gyrus/insula area, extending to the orbitofrontal gyrus. In other words, as age increased, activation in these areas during inhibitory control increased. A limitation of this work, however, was that a blocked design rather than an event-related design was utilized (Henson, 2006). Thus, activation reflected a mixture of regions involved in the task and possibly regions involved in error processing, conflict processing, response preparation, response state (set), and stimulus analysis. Furthermore, with the experimental design used by these authors, identifying activation elicited purely by No-go trials was not possible because the experimental blocks were a mixture of Go and No-go trials. This limitation in their study design may be the reason for the lack of replication of prior adult findings in this experiment such as the findings reported by Liddle et al. (2001) and Watanabe et al. (2002).

In a more recent study, Stevens, Kiehl, Pearlson, $\&$ Calhoun (2007) used a Go/No-go task to investigate the functional neural networks that support inhibitory control in 50 healthy participants who were grouped by age into adolescents (11-17 years old; mean age $=14.7$ years; $\mathrm{SD}=2.0$ years) and adults $(18-37$ years old; mean age $=25.1$ years; $\mathrm{SD}=5.7$ years) with no significant differences in the gender proportion between adolescents and adults. They first identified functionally connected regions wherein activation was elicited by the Go/No-go task using a multivariate analysis method (i.e., independent component analysis) that identified brain regions with similar temporal patterns of signal changes. Dynamic causal modeling (Friston, Harrison, \& Penny, 2003) was then applied to these regions to identify their influences on each other (i.e., effective connectivity). The authors found that response inhibition in this task was led by the control exerted by fronto-striatal-thalamic networks over parietal-premotor networks. When the demand for response inhibition increased, fronto-striatalthalamic circuits released parietal-premotor networks from their control, resulting in greater engagement of the latter regions in performance on the Go/No-go task. Compared with adults, adolescents exhibited diminished integration between the regions that comprised the fronto-striatal-thalamic network, an aspect that was associated with decreased behavioral performance. This finding was interpreted as evidence that these regions are less specialized in inhibitory control during adolescence compared with adulthood.

These findings are consistent with the study reported by Rubia et al. (2006) in which a Go/No-go task was also used to investigate inhibitory control in 23 adults (20-43 years old; mean age $=28.0$ years; SD $=6.0$ years) and 25 adolescents $(10-17$ years old; mean age $=15.0$ years; $\mathrm{SD}=2.0$ years). Raven's Standard Progressive Matrices Intelligence Questionnaire (Raven, 1960) revealed between-group differences. Analyses of covariance were then conducted with IQ as the covariate to determine group differences in the $\mathrm{Go} /$ No-go performance measures. Similar to the findings reported by Stevens, Kiehl, Pearlson, \& Calhoun (2007), Rubia et al. (2006) found that adults exhibited an increase in activation compared with adolescents in fronto-striatal regions including the anterior cingulate gyrus and caudate. These differences between adolescents and adults were interpreted as reflecting the protracted maturation of fronto-striatal networks that are engaged during inhibitory control.

The study reported by Tamm et al. (2002) found increased activation across development in frontal regions that are often involved in cognitive control such as the left inferior frontal gyrus and orbitofrontal gyrus; however, this study adopted a blocked design that hindered stronger interpretations of these findings. Nevertheless, as demonstrated by Rubia et al. (2006) and Stevens, Kiehl, Pearlson, \& Calhoun (2007), frontostriatal regions such as the anterior cingulate and caudate appear to play an important role in response inhibition during performance of the Go/No-go task, and the participation of these regions appears to significantly increase the inhibition of undesired responses as individuals become older.

In addition to studies that utilized anti-saccade and Go/No-go tasks, by the time the present review was prepared for submission, one fMRI study that used the Stroop task (Adleman et al., 2002) and one that used a stop-signal task (Rubia, Smith, Taylor, \& Brammer, 2007) to investigate inhibitory control in adolescents were available in the literature. The study that used the Stroop task analyzed 11 adolescents (12.6-16.8 years old; mean age $=14.7$ years; $\mathrm{SD}=1.3$ years; seven females) and 11 adults (17.4-22.7 years old; mean age $=20.0$ years; $\mathrm{SD}=1.7$ years). This study included only individuals with a full-scale IQ $>80$ measured by the WISC-III and WAIS-III. They found that adolescents and young adults exhibited similar involvement of parietal regions while performing the task, although adults exhibited an increase in activation of the left middle frontal gyrus compared with adolescents (Adleman et al., 2002). The blocked design used by these authors, however, precluded a strong interpretation of these data. The study that utilized a stop-signal task analyzed 26 adolescents (10-17 years old; mean age $=15.0$ years; $\mathrm{SD}=2.0$ years $)$ and 21 adults $(20-42$ years old; mean age $=28.0$ years; $\mathrm{SD}=5.0$ years). All participants were male, and IQ was measured using Raven's Standard Progressive Matrices Intelligence Questionnaire (Raven, 1960). IQ scores were entered as a covariate in the analysis. Activation in the bilateral insula, left thalamus, putamen, and posterior cingulate 
gyrus were negatively correlated with age, a finding that may indicate compensatory mechanisms. Young adults in this study also exhibited greater activation in the right inferior prefrontal cortex during successful inhibition and rostral anterior cingulate gyrus during inhibition failure compared with adolescents. The age range of the adolescents in this study, however, was 10-17 years, comprising an excessively variable sample in contrast to the other studies reported in the present review (see Rubia et al., 2006), a fact that can hinder the interpretation of the data.

\section{Discussion}

The studies reviewed herein demonstrate that the performance of inhibitory control in adolescents engages regions that are typically involved in inhibitory control in adults such as the dorsal anterior cingulate cortex (Velanova et al., 2008), the dorsolateral prefrontal cortex (Velanova et al., 2009; Luna et al., 2001), and fronto-striatal regions (Rubia et al., 2006; Stevens, Kiehl, Pearlson, \& Calhoun, 2007). In contrast to adults, however, adolescents exhibited a decrease in activation in these regions, a finding that can be interpreted as reflecting the protracted development of these regions during this developmental period (Gogtay et al., 2004). Furthermore, investigations of the functional connectivity between regions involved in inhibitory control suggest that adolescents, in contrast to adults, exhibit reduced functional connectivity from frontal regions to other brain regions (both cortical and subcortical; Hwang et al., 2010) and from frontostriatal-thalamic networks to parietal-premotor networks (Stevens, Kiehl, Pearlson, \& Calhoun, 2007). The impaired connectivity between these regions in adolescents, in addition to the aforementioned diminished activity of distinct prefrontal regions in this population, may be a likely cause of the inhibitory control limitations found in this developmental period.

The two experiments in which reward was provided (Padmanabhan et al., 2011; Geier et al., 2010) suggest that the ventral striatum plays an important role in reward processing during the exertion of inhibitory control, both in adults and adolescents. As reported by Geier et al. (2010), this region exhibited a reduction of activity in adolescents compared with adults during the assessment of reward cues, suggesting that adolescents do not process reward cues as thoroughly as adults. During the preparation to respond to rewarded trials, however, adolescents exhibited greater activity in this region compared with adults, a finding interpreted as enhanced reactivity to the expectancy of the forthcoming reward (Geier et al., 2010). Unfortunately, Padmanabhan et al. (2011) did not report analyses of the preparation and assessment of cues as did Geier et al. (2010), precluding the verification of whether the findings reported by Geier et al. (2010) are replicable.

Despite the potential benefits of a broader understanding of the peculiarities of the healthy adolescent brain (Insel, 2010), few fMRI studies have investigated this issue utilizing inhibitory control tasks. Only 11 empirical articles that investigated this issue were found in the selected databases by the time this article was submitted and revised. Although these are highly informative studies, more research is necessary to elucidate the role of specific regions in the performance of inhibitory control in adolescents such as the dorsolateral cortex, which was clearly less activated in adolescents compared with adults in two experiments (Velanova et al., 2009; Luna et al., 2001), but apparently not in the other nine reports.

Inhibitory control, together with other cognitive control functions, is deeply entangled with other cognitive processes (Badre \& Wagner, 2004). Future research would benefit from the development of tasks in which interactions between inhibitory control and other cognitive functions can be investigated in adolescents. An example of such an approach would be to develop experimental paradigms to examine memory performance as a function of different levels of cognitive control (Jaeger, Cox, \& Dobbins, 2012; Jaeger, Selmeczy, O'Connor, Diaz, \& Dobbins, 2012; Ghetti, DeMaster, Yonelinas, \& Bunge, 2010). Another research possibility in adolescents would be the investigation of inhibitory control for emotional information or the influence of emotional state on inhibitory control abilities (Ochsner \& Gross, 2005). To date, these issues have not been studied in this population, but such findings could be a valuable addition to the current knowledge on the functional organization of the adolescent brain.

\section{Acknowledgements}

This research was supported by Fundação de Amparo à Pesquisa do Estado de São Paulo (FAPESP).

\section{References}

Adleman, N. E., Menon, V., Blasey, C. M., White, C. D., Warsofsky, I. S., Glover, G. H., \& Reiss, A. L. (2002). A developmental fMRI study of the Stroop color-word task. Neurolmage, 16, 61-75.

Badre, D., \& Wagner, A. D. (2004). Selection, integration, and conflict monitoring: Assessing the nature and generality of prefrontal cognitive control mechanisms. Neuron, 41, 473-487.

Bjork, J. M., Knutson, B., Fong, G. W., Caggiano, D. M., Bennett, S. M., \& Hommer, D. W. (2004). Incentive-elicited brain activation in adolescents: Similarities and differences from young adults. Journal of Neuroscience, 24, 1793-1802.

Botvinick, M. M., Braver, T. S., Barch, D. M., Carter, C. S., \& Cohen, J. D. (2001). Conflict monitoring and cognitive control. Psychological Review, 108, 624-652.

Casey, B. J., Getz, S., \& Galvan, A. (2008). The adolescent brain. Development Review, 28, 62-77.

Casey, B. J., \& Jones, R. M. (2010). Neurobiology of the adolescent brain and behavior: Implications for substance use disorders. Journal of the American Academy of Child and Adolescent Psychiatry, 49, 1189-1201.

Colby, C. L., \& Goldberg, M. E. (1999). Space and attention in parietal cortex. Annual Review of Neuroscience, 22, 319-349.

Corbetta, M., \& Shulman, G. L. (2002). Control of goal-directed and stimulus-driven attention in the brain. Nature Reviews Neuroscience, 3, 201-215.

Delgado, M. R., Locke, H. M., Stenger, V. A., \& Fiez, J. A. (2003). Dorsal striatum responses to reward and punishment: Effects of valence and magnitude manipulations. Cognitive, Affective, \& Behavioral Neuroscience, 3, 27-38.

Eaton, D. K., Kann, L., Kinchen, S., Ross, J., Hawkins, J., Harris, W. A., ... Wechsler, H. (2006). Youth risk behavior surveillance: United States, 2005. Morbidity and Mortality Weekly Report, 55, 1-108. 
Everling, S., \& Munoz, D. P. (2000). Neuronal correlates for preparatory set associated with pro-saccades and anti-saccades in the primate eye field. Journal of Neuroscience, 20, 387-400.

Falkenstein, M., Hoorman, J., \& Hohnsbein, J. (1999). ERP components in Go/Nogo tasks and their relation to inhibition. Acta Psychologica, 101, 267-291.

Fischer, B., Biscaldi, M., \& Gezeck, S. (1997). On the development of voluntary and reflexive components in human saccade generation. Brain Research, 754, 285-297.

Friston, K. J., Harrison, L., \& Penny, W. (2003). Dynamic causal modelling. NeuroImage, 19, 1273-1302.

Garavan, H., Ross, T. J., \& Stein, E. A. (1999). Right hemispheric dominance of inhibitory control: An event-related functional MRI study. Proceedings of the National Academy of Sciences of the United States of America, 96, 8301-8306.

Geier, C. F., Terwilliger, R., Teslovich, T., Velanova, K., \& Luna, B. (2010). Immaturities in reward processing and its influence on inhibitory control in adolescence. Cerebral Cortex, 20, 1613-1629.

Ghetti, S., DeMaster, D. M., Yonelinas, A. P., \& Bunge, S. A. (2010). Developmental differences in medial temporal lobe function during memory encoding. Journal of Neuroscience, 30, 9548-9556.

Gogtay, N., Giedd, J. N., Lusk, L., Hayashi, K. M., Greenstein, D., Vaituzis, A. C., ... Thompson, P. M. (2004). Dynamic mapping of human cortical development during childhood through early adulthood. Proceedings of the National Academy of Sciences of the United States of America, 101, 8174-8179.

Henson, R. N. A. (2006). Efficient experimental design for fMRI. In K. J. Friston, J. T. Ashburner, S. J. Kiebel, T. E. Nichols, \& W. D. Penny (Eds.), Statistical parametric mapping: The analysis of functional brain images (pp. 193-210). Amsterdam: Elsevier.

Huster, R. J., Westerhausen, R., Pantev, C., \& Konrad, C. (2010). The role of the cingulate cortex as neural generator of the N200 and P300 in a tactile response inhibition task. Human Brain Mapping, $31,1260-1271$.

Hwang, K., Velanova, K., \& Luna, B. (2010). Strengthening of top-down frontal cognitive control networks underlying the development of inhibitory control: A functional magnetic resonance imaging effective connectivity study. Journal of Neuroscience, 30 , 15535-15545.

Insel, T. R. (2010). Rethinking schizophrenia. Nature, 468, 187-193.

Jaeger, A., Cox, J. J., \& Dobbins, I. G. (2012). Recognition confidence under violated and confirmed memory expectations. Journal of Experimental Psychology: General, 141, 282-301.

Jaeger, A., Selmeczy, D., O'Connor, A. R., Diaz, M., \& Dobbins, I. G. (2012). Prefrontal cortex contributions to controlled memory judgment: fMRI evidence from adolescents and young adults. Neuropsychologia, 50, 3745-3756.

Liddle, P. F., Kiehl, K. A., \& Smith, A. M. (2001). Event-related fMRI study of response inhibition. Human Brain Mapping, 12, 100-109.

Luck, S. J. (2005). An introduction to the event-related potential technique. Cambridge: MIT Press.

Luna, B., Garver, K. E., Urban, T. A., Lazar, N. A., \& Sweeney, J. A. (2004). Maturation of cognitive processes from late childhood to adulthood. Child Development, 75, 1357-1372.

Luna, B., Padmanabhan, A., \& O'Hearn, K. (2010). What has fMRI told us about the development of cognitive control during adolescence? Brain and Cognition, 72, 101-113.

Luna, B., \& Sweeney, J. A. (2004). The emergence of collaborative brain function: fMRI studies of the development of response inhibition. Annals of the New York Academy of Sciences, 1021, 296-309.

Luna, B., Thulborn, K. R., Munoz, D. P., Merriam, E. P., Garver, K. E., Minshew, N. J., ... Sweeney, J.A. (2001). Maturation of widely distributed brain function subserves cognitive development. NeuroImage, 13, 786-793.

MacDonald, A. W., 3rd, Cohen, J. D., Stenger, V. A., Carter, C. S. (2000). Dissociating the role of the dorsolateral prefrontal and anterior cingulate cortex in cognitive control. Science, 288, 1835-1838.

Miller, E. K. (2000). The prefrontal cortex and cognitive control. Nature Reviews Neuroscience, 1, 59-65.

Munoz, D. P., Broughton, J. R., Goldring, J. E., \& Armstrong, I. T. (1998). Age-related performance of human subjects on saccadic eye movement tasks. Experimental Brain Research, 121, 391-400.

Munoz, D. P., \& Everling, S. (2004). Look away: The anti-saccade task and the voluntary control of eye movement. Nature Reviews Neuroscience, 5, 218-228.
Nieuwenhuis, S., Yeung, N., van den Wildenberg, W., \& Ridderinkhof, K. R. (2003). Eletrophysiological correlates of anterior cingulate function in a go/no-go task: Effects of reponse conflict and trial type frequency. Cognitive, Affective, \& Behavioral Neuroscience, $3,17-26$.

Ochsner, K. N., \& Gross, J. J. (2005). The cognitive control of emotion. Trends in Cognitive Sciences, 9, 242-249.

O'Driscoll, G. A., Alpert, N. M., Matthysse, S. W., Levy, D. L., Rauch, S. L., \& Holzman, P. S. (1995). Functional neuroanatomy of antisaccade eye movements investigated with positron emission tomography. Proceedings of the National Academy of Sciences of the United States of America, 92, 925-929.

Padmanabhan, A., Geier, C. F., Ordaz, S. J., Teslovich, T., \& Luna, B. (2011). Developmental changes in brain function underlying the influence of reward processing on inhibitory control. Developmental Cognitive Neuroscience, 1, 517-529.

Pierrot-Deseilligny, C., Muri, R. M., Nyffeler, T., \& Milea, D. (2006). The role of the human dorsolateral prefrontal cortex in ocular motor behavior. Annals of the New York Academy of Sciences, 1039, 239-251.

Pierrot-Deseilligny, C., Rivaud, S., Gaymard, B., Muri, R., \& Vermersch, A. I. (1995). Cortical control of saccades. Annals of Neurology, 37, 557-567.

Polli, F. E., Barton, J. J. S., Cain, M. S., Thakkar, K. N., Rauch, S. L., \& Manoach, D. S. (2005). Rostral and dorsal anterior cingulate cortex make dissociable contributions during antisaccade error commission. Proceedings of the National Academy of Sciences of the United States of America, 102, 15700-15705.

Raven, J. C. (1960). Guide to the standard progressive matrices: Sets $A, B, C, D$ and $E$. London: HK Lewis.

Ridderinkhof, K. R., Ullsperger, M., Crone, E., \& Nieuwenhuis, S. (2004). The role of the medial frontal cortex in cognitive control. Science, 306, 443-447.

Roebroeck, A., Formisano, E., \& Goebel, R. (2005). Mapping directed influence over the brain using Granger causality and fMRI. NeuroImage, 25, 230-242.

Rubia, K., Smith, A. B., Taylor, E., \& Brammer, M. (2007). Linear age-correlated functional development of right inferior frontostriato-cerebellar networks during response inhibition and anterior cingulate during error-related processes. Human Brain Mapping, $28,1163-1177$.

Rubia, K., Smith, A. B., Woolley, J., Nosarti, C., Heyman, I., Taylor, E., \& Brammer, M. (2006). Progressive increase of frontostriatal brain activation from childhood to adulthood during event-related tasks of cognitive control. Human Brain Mapping, 27, 973-993.

Simmonds, D. J., Pekar, J. J., \& Mostofsky, S. H. (2008). Meta-analysis of Go/No-go tasks demonstrating that fMRI activation associated with response inhibition is task-dependent. Neuropsychologia, 46, 224-232.

Stevens, M. C., Kiehl, K. A., Pearlson, G. D., \& Calhoun, V. D. (2007). Functional neural networks underlying response inhibition in adolescents and adults. Behavioral Brain Research, 181, 12-22.

Stuphorn, V., Taylor, T. L., \& Schall, J. D. (2000). Performance monitoring by the supplementary eye field. Nature, 408, 857-860.

Tamm, L., Menon, V., \& Reiss, A. L. (2002). Maturation of brain function associated with response inhibition. Journal of the American Academy of Child \& Adolescent Psychiatry, 41, 12311238.

Trappenberg, T. P., Dorris, M. C., Munoz, D. P., \& Klein, R. M. (2001). A model of saccade initiation based on the competitive integration of exogenous and endogenous signals in the superior colliculus. Journal of Cognitive Neuroscience, 13, 256-271.

Velanova, K., Wheeler, M. E., \& Luna, B. (2009). The maturation of task set-related activation supports late developmental improvements in inhibitory control. Journal of Neuroscience, 29, 12558-12567.

Velanova, K., Wheeler, M. E., \& Luna, B. (2008). Maturational changes in anterior cingulate and frontoparietal recruitment support the development of error processing and inhibitory control. Cerebral Cortex, 18, 2505-2522.

Watanabe, J., Sugiura, M., Sato, K., Sato, Y., Maeda, Y., Matsue, Y., ... Kawashima, R. (2002). The human prefrontal and parietal association cortices are involved in NO-GO performances: An event-related fMRI study. NeuroImage, 17, 1207-1216.

Wechsler D (1999). WASI: Wechsler Abbreviated Scale of Intelligence. San Antonio, TX: Psychological Corporation. 